\section{TPACKPEC: DISEÑO DE SITUACIONES DE APRENDIZAJE MEDIADAS POR TIC EN EDUCACIÓN FÍSICA}

\author{
TPACKPEC: DESIGN OF ICT-MEDIATED LEARNING SITUATIONS IN PHYSICAL \\ EDUCATION
}
TPACKPEC: DESENHO DE SITUAÇÕES DE APRENDIZAGEM MEDIADAS POR TIC EM EDUCAÇÃO FÍSICA

\author{
Meritxell Monguillot Hernando*, Montse Guitert Catasús*, \\ Carles González Arévalo*
}

\begin{abstract}
Palabras clave:
TIC.

Tecnología de la información. Comportamiento cooperativo. Colaboración. Emociones.

Resumen: El presente estudio tiene como objetivo identificar cuáles son los elementos clave para diseñar situaciones de aprendizaje mediadas por las TIC en Educación Física a través de la colaboración docente. Para ello, se ha seguido el modelo de investigación basada en diseño (IBD) en la que han participado tres docentes y cuatro centros educativos. Las iteracciones entre profesores e investigadores ha resultado en una evolución continua de los elementos clave, llevando a una tipología de conocimiento con cuatro categorías: el modelo TPACKPEC. Este modelo es una evolución del TPACK (KOEHLER; MISHRA, 2009) ya que no solo integra tecnología, pedagogía y currículo en las aulas, sino que también introduce las competencias emocionales como elementos fundamentales en el proceso de enseñanza y aprendizaje.
\end{abstract}

Palavras chave: TIC.

Tecnologia de informação. Comportamento cooperativo. Colaboração. Emoções.

Keywords:

ICT. Information technology. Cooperative behaviour. llaboration Emotions

Resumo: 0 presente estudo tem como objetivo identificar quais os principais elementos para a concepção de situações de aprendizagem mediadas pelas TIC na Educação Física através da colaboração docente. Para tal, seguiu-se o modelo de pesquisa baseada em design (IBD), na qual participaram três professores e quatro centros educacionais. As iterações entre professores e pesquisadores resultou numa evolução contínua dos elementos-chave, dando origem a uma tipologia de conhecimento com quatro categorias: o modelo TPACKPEC. Este modelo é uma evolução do TPACK (KOEHLER; MISHRA, 2009) porque não só integra tecnologia, pedagogia e currículo na sala de aula, mas também apresenta competências emocionais como elementos fundamentais no processo de ensino e aprendizagem.

Abstract: This study points out the key elements for designing ICT-mediated learning situations in Physical Education through educational collaboration. It has followed a Design-Based Research method in which three teachers and four educational centers have participated. As a result of the iterations carried out by the teachers and the researcher, a continuous evolution of the key elements led us to classify them in four categories of knowledge resulting in the TPACKPEC model. This model evolves from the TPACK model (KOEHLER; MISHRA, 2009) as it not only integrates technology, pedagogy and curriculum in the classroom but also introduces emotional competences as key elements in the teaching and learning process...
*Universitat Oberta de Catalunya (UOC) E-mail: mmonguillot@uoc.edu; mguitert@uoc.edu; cargonzalez@gencat.cat

Recebido em: 23-09-2017 Aprovado em: 04-06-2018

DOI: https://doi.org/10.22456/1982-8918.76681 (c) (1) (8) Licence 


\section{INTRODUCCIÓN}

La sociedad del conocimiento vive inmersa en cambios sociales, económicos y tecnológicos que obligan a repensar y cambiar el rol educativo. La tecnología está presente en todos los ámbitos de la vida y forma parte de la realidad del alumnado. La escuela no puede quedarse atrás y debe incorporarla en el proceso de enseñanza y aprendizaje (GARCÍA, 2011). No obstante, se hace necesario replantear la integración de las tecnologías de la información y comunicación (en adelante TIC) en el aula a fin de ir más allá del mero contenido curricular y del uso de determinadas herramientas tecnológicas (GALLEGO et al., 2017). En esta línea, el presente estudio toma de referencia como marco metodológico para integrar la tecnología en el aula el modelo TPACK (KOEHLER; MISHRA, 2009). Este enfoque otorga la misma importancia a la tecnología, pedagogía y contenido disciplinar, enfatizando la importancia de la interrelación entre los tres aspectos (DURALL et al., 2012).Adoptar el modelo TPACK en la docencia conlleva integrar la tecnología como un aspecto clave a la hora de planificar e implementar la enseñanza alejándose del enfoque centrado solamente en el uso de la tecnología y avanzando hacia la incorporación de ésta a partir de la integración pedagógica y curricular (DURALL et al., 2012).

Además, el presente estudio integra las recientes aportaciones de la neurociencia al ámbito educativo que invitan a atender al desarrollo emocional del alumnado como factores fundamentales para mejorar el aprendizaje. Para conseguirlo, las emociones son elementos clave que deben estar presentes en el aula si queremos mejorar los procesos educativos y hacer que el aprendizaje deje huella en el alumnado (MORA, 2013; IBARROLA, 2013).

De esta manera, el objetivo general del estudio ha sido identificar los elementos clave para diseñar situaciones de aprendizaje mediadas por TIC en Educación Física a través de la colaboración docente.

\subsection{El modelo TPACK: integrando tecnología, pedagogía y currículo en el aula}

El modelo TPACK (Techonological Pedagogical Content Knowledge) creado por Koehler y Mishra (2009) presenta un marco metodológico que relaciona la tecnología, la pedagogía y el conocimiento disciplinar o curricular, para que el conocimiento enseñado y aprendido sea eficiente. Según múltiples autores (KOEHLER; MISHRA, 2009; VALVERDE et al., 2010; CABERO et al. 2014; HERNANDO, 2015; DURALL et al., 2012) el modelo TPACK se basa en la intersección de tres componentes que forman parte del conocimiento docente: el conocimiento curricular (CK) propio de la materia a impartir, el conocimiento pedagógico (PK) que permite entender el proceso de enseñanza y aprendizaje, y el conocimiento tecnológico (TK) que posibilita utilizar las TIC para múltiples tareas. Los tres conocimientos están interrelacionados y generan tres nuevos dominios: el conocimiento pedagógico disciplinar, el conocimiento tecnológico disciplinar y el conocimiento tecnopedagógico (DURALL et al., 2012).

El ámbito tecnológico se relaciona con los programas y dispositivos móviles (TK), el ámbito pedagógico se vincula con el saber didáctico y los métodos de enseñanza (PK), mientras que el ámbito disciplinar es el conocimiento de cada materia (CK) (HERNANDO, 2015). En esta línea, Valverde et al. (2010) sostienen que la actividad docente requiere de los tres tipos de conocimiento y una buena práctica que integre las TIC en el aula debe incluir los tres componentes. Salinas et al. (2014) defienden que los nuevos escenarios de aprendizaje 
requieren nuevas competencias docentes y que el modelo TPACK contribuye a la mejora de estas competencias asociadas al uso de las TIC. De acuerdo con este autor, el conocimiento disciplinar (CK) o curricular es el conocimiento sobre una materia que se enseña y aprende. El conocimiento pedagógico (PK) es el conocimiento sobre los procesos, estrategias y métodos de enseñanza y aprendizaje y engloba los objetivos de aprendizaje, los valores, la gestión escolar, la planificación docente, el desarrollo curricular y la evaluación de los aprendizajes. Finalmente, el conocimiento tecnológico (TK) está en constante cambio, más que los otros dos conocimientos, y permite realizar una tarea mediante distintas herramientas TIC.

La integración de todos los elementos genera el modelo TPACK que Koehler y Mishra (2009) defienden como una nueva forma de conocimiento que va más allá de los tres componentes y que es la base de una buena práctica educativa que integra las TIC. Incorporar la tecnología en la docencia supone relacionar el conocimiento disciplinar y tecnológico mediante un uso de las TIC centrado en la colaboración entre participantes búsqueda de información, publicación y creación de materiales. La propuesta metodológica de Koehler y Mishra (2009) permite avanzar hacia la incorporación de la tecnología a partir de la integración pedagógica y curricular (DURALL et al., 2012).

Figura 1 - Modelo TPACK

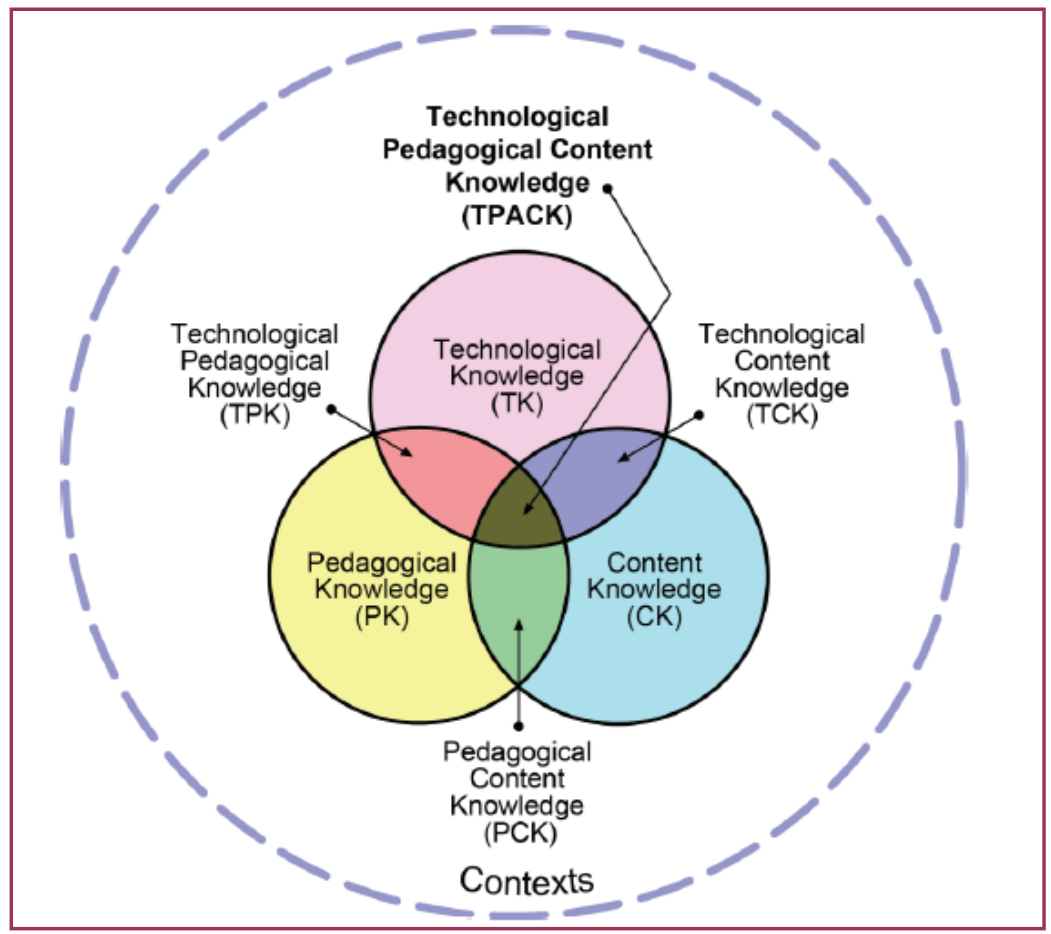

Fuente: TPACK, 2012

\subsection{Una educación física saludable y mediada por tic}

Gran parte de la sociedad lleva un estilo de vida sedentario y los adolescentes no son excepción (PIÉRON et al., 2008). Recientes estudios demuestran como la obesidad infantil es cada vez más frecuente en la infancia siendo niños y jóvenes un grupo de riesgo sobre el que incidir y actuar en la prevención. La inactividad física es el cuarto factor de riesgo más importante de mortalidad en el mundo (un $6 \%$ de defunciones a nivel mundial) y es superado por la hipertensión, el exceso de glucosa en sangre, el consumo de tabaco, la obesidad y el 
sobrepeso (ORGANIZACIÓN MUNDIAL DE LA SALUD, 2010). En esta línea, la Educación Física se centra en la adquisición de hábitos de vida saludable pero puede ser mediada por la tecnología de forma intencional y crítica. A pesar que las TIC puedan ser vistas, en un primer momento, como amenazas de la Educación Física y se considere que puedan restar minutos de práctica motriz, tienen un gran potencial. Por ejemplo, como complemento a la práctica deportiva mediante el uso de webs y blogs, o bien, como herramientas de interacción y monitorización de la práctica física (CAPLLONCH, 2005; LLEIXÀ, 2007; GONZÁLEZ et al., 2014).

Ferreres (2011) y Tirado (2010) señalan que ante una sociedad cada vez más tecnológica, la Educación Física, a pesar de su carácter práctico, no puede quedarse impasible y debe adecuarse a los nuevos cambios sociales que aportan las TIC. Los beneficios de las TIC para la materia son múltiples, son una fuente de información, se convierten en una potente herramienta de comunicación e intercambio de experiencias y materiales didácticos para el profesorado abriendo nuevas formas de colaborar, aprender y trabajar que el docente de Educación Física no puede desaprovechar (FERRERES, 2011; GONZÁLEZ, et al., 2016;). En esta línea, se observa como las TIC facilitan la colaboración virtual docente y la creación conjunta de conocimiento curricular (MONGUILLOT et al., 2013), y favorecen el acceso a la información ayudando al alumnado a organizar y planificar su propia actividad física (LLEIXÀ, 2007). Esta última idea posiciona al m-learning o aprendizaje móvil como una herramienta de aprendizaje emergente en el aula de Educación Física (MONGUILLOT et al., 2014). Coincidiendo con la visión de autores como Tirado (2010), Prat y Camerino (2013) y González et al. (2014) las situaciones de la investigación utilizan las TIC como herramienta pedagógica para facilitar el aprendizaje autónomo e incentivar la práctica de actividad física en el alumnado. De este modo, los objetivos de aprendizaje de las situaciones son los que marcan el uso de las TIC y orientan qué herramientas seleccionar y por qué (CABERO et al., 2014). De acuerdo con este argumento, las herramientas TIC seleccionadas en la investigación responden a la finalidad e intencionalidad de cada situación de aprendizaje.

\subsection{Aportes de la neurociencia a la educación}

La neurociencia entendida como la ciencia que estudia el funcionamiento del cerebro, está demostrando la relevancia que tiene para los docentes saber cómo funciona y aprende el cerebro para la mejora del aprendizaje. La neurociencia muestra la importancia para el aprendizaje de atender a ciertos aspectos que son relevantes para el cerebro que aprende, entre ellos se encuentra la atención, la curiosidad, las emociones, la conciencia, la memoria, los procesos mentales, el sueño y los ritmos biológicos. Junto al término de neurociencia aparece el de neuroeducación entendido como un nuevo enfoque de la enseñanza que estudia los conocimientos del cerebro e integra los saberes de la psicología, sociología y medicina para la mejora de los procesos de enseñanza aprendizaje. La neuroeducación aplica en el aula los conocimientos sobre las emociones, la curiosidad y la atención a fin de mejorar los procesos de enseñanza de los profesores y el aprendizaje de los estudiantes (MORA, 2013; IBARROLA, 2013). En esta línea, las situaciones de la investigación han potenciado aprendizajes vivenciales, emocionantes, basados en el juego, el asombro, y la sorpresa mediante la introducción de retos, desafíos, acertijos y códigos secretos que han despertado la curiosidad del alumnado y mejorado el aprendizaje. 


\section{MÉTODO}

\subsection{Metodología}

La pregunta que ha guiado el estudio ha sido: ¿Cuáles son los elementos clave para diseñar una situación de aprendizaje mediada por TIC en Educación Física? y para responder a la pregunta se ha utilizado la investigación basada en el diseño (en adelante IBD) entendida como un paradigma de investigación emergente cuya finalidad es diseñar y explorar innovaciones educativas mediante la investigación e identificación de las características relevantes del diseño y su resultado. La IBD es un diseño iterativo dirigido a intervenir, pero fundamentado por la teoría (GROS, 2012). La finalidad última de las investigaciones centradas en el diseño es la creación de contribuciones teóricas ya sea para extender o modificar la teoría existente o para generar nueva teoría (AMIEL; REEVES, 2008; GIBELLI, 2014). En educación la IBD está cobrando un especial interés ya que pretende aportar mejoras educativas estudiando contextos naturales a fin de crear modificaciones que mejoren el aprendizaje y la práctica educativa (GIBELLI, 2014; GROS, 2012). La IBD enfatiza la iteración continua entre participantes no solo para construir un producto sino para mejorar sistemáticamente la innovación mientras produce principios de diseño que puedan ser útiles para otros contextos similares (AMIEL; REEVES, 2008). La IBD se caracteriza por la necesidad de ubicar la investigación en un contexto real y por generar cambios específicos en dicho contexto mediante un diseño sistémico, iterativo y cíclico (GIBELLI, 2014).

Las investigaciones que se basan en la IBD siguen una serie de pasos iterativos y cíclicos para su monitorización que apuntan a unas fases de análisis del contexto, diseño, implementación y evaluación (RINAUDO; DONOLO, 2010; GROS, 2012; GIBELLI, 2014). En la presente investigación, se ha aplicado la IBD para descubrir cuáles son los elementos clave para diseñar situaciones de aprendizaje en Educación Física mediadas por TIC a través de la colaboración docente. Para hacerlo y basándonos en las propuestas de Gros (2012) y Gibelli (2014) la IBD se ha articulado en dos fases: de análisis y modificación, y de aplicación y revisión. Ambas fases han aglutinado los pasos de diseño, implementación y evaluación de los elementos clave que se han monitorizado de forma colaborativa virtual. La siguiente figura muestra el ciclo de fases de la IBD en la investigación.

Figura 2 - Ciclo de las fases de la IBD en la investigación

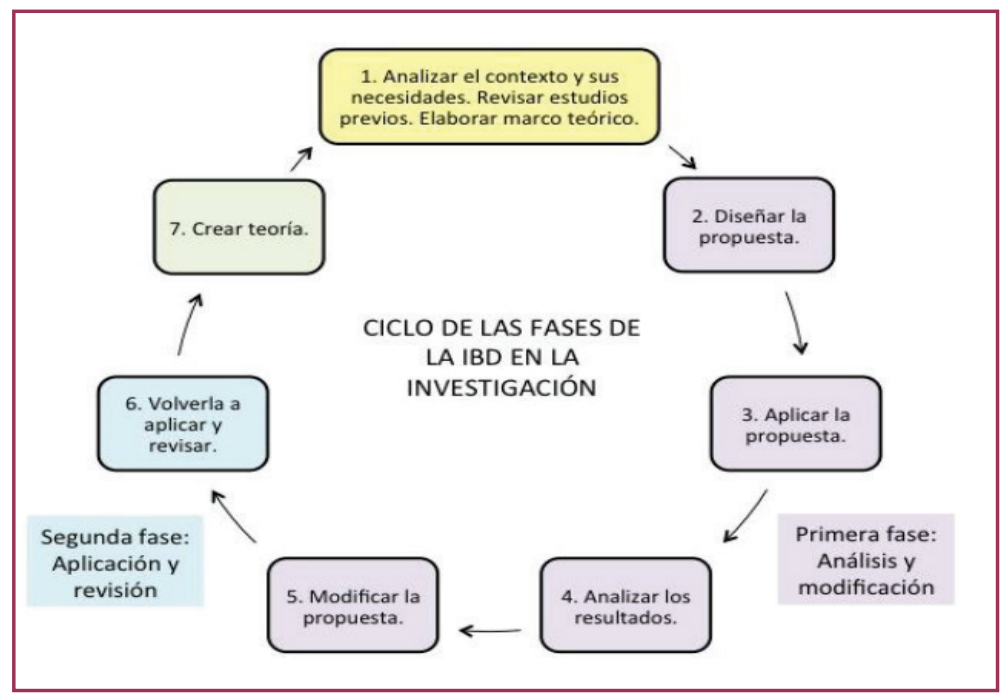

Fuente: Elaboración propia. 


\subsection{Escenarios de la investigación}

En la investigación han participado tres profesores de Educación Física de Educación Secundaria Obligatoria siendo uno de ellos la investigadora. En total han participado cuatro centros educativos de Barcelona ciudad de distinta tipología de los cuales son docentes los profesores seleccionados. En esta línea, la investigadora ha participado replicando las condiciones de las situaciones en su propia aula como el resto de docentes, y ha guiado en las fases de diseño, implementación y evaluación a los docentes mediante la colaboración e interacción constante (HERNÁNDEZ, 2015).

Una de las características de la investigación ha sido el trabajo colaborativo virtual realizado entre los docentes y la investigadora mediante la IBD, que ha propiciado una interacción y comunicación intensa generando cambios en el contexto y conocimiento. De hecho, los docentes han participado en la toma de decisiones junto a la investigadora en las distintas fases del estudio y durante 2 años.

Figura 4 - Docentes, centros participantes y herramientas

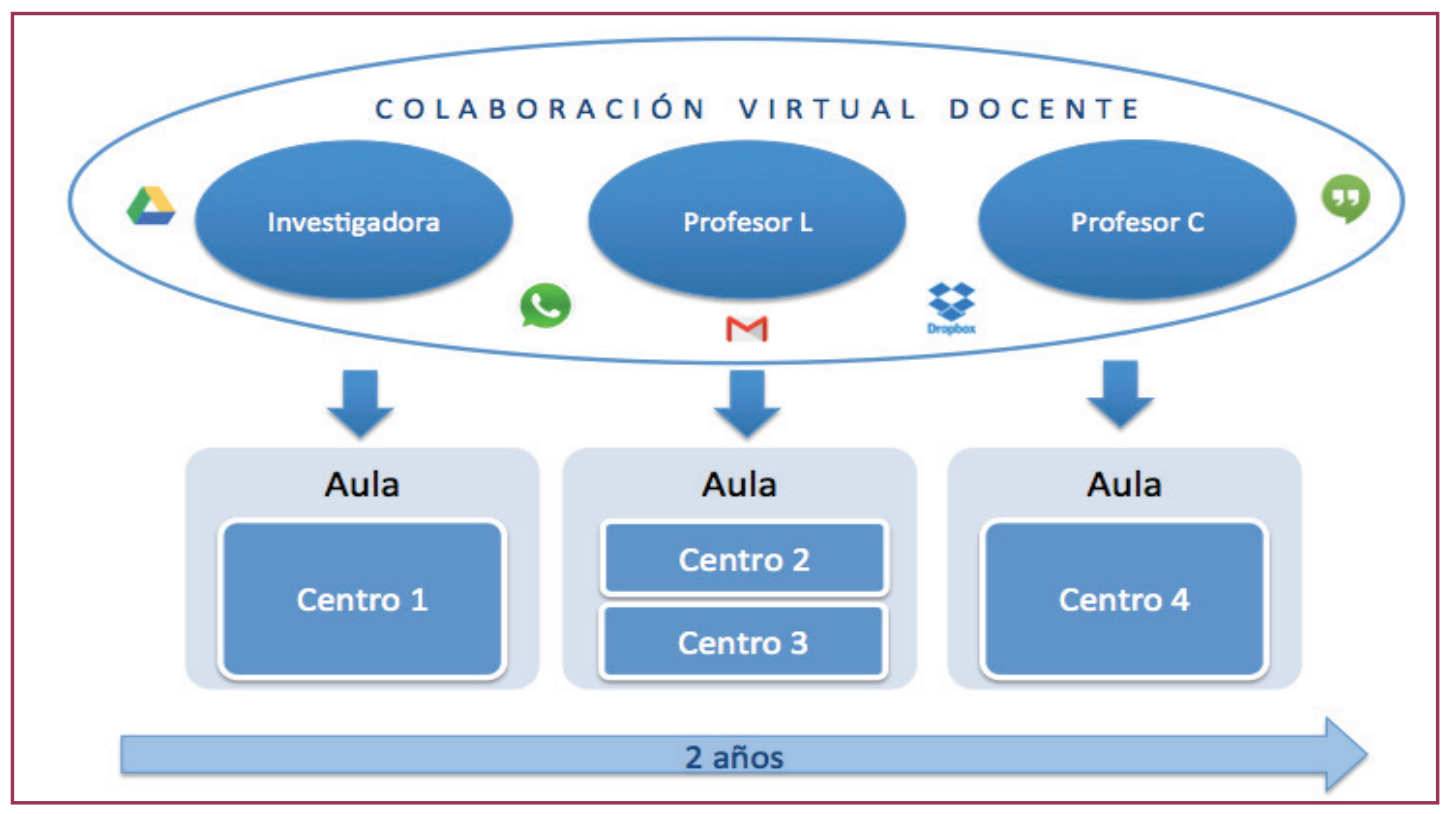

Fuente: Elaboración propia.

\subsection{Instrumentos}

Los instrumentos utilizados a lo largo del estudio han variado en función del objetivo y de la fase de la investigación. Para el diseño de las situaciones de aprendizaje que han integrado los elementos clave se han utilizado los focus group presenciales entre la investigadora y el profesorado, y el análisis de documentos curriculares. Para el seguimiento de la implementación de los elementos clave en las situaciones de aprendizaje en el aula, se ha utilizado la observación participante mediante el uso de un grupo de WhatsApp (MONGUILLOT et al., 2017) que ha tomado forma de diario colaborativo virtual mediante el cual el profesorado ha compartido información diaria sobre la implementación de los elementos en el aula. Para la evaluación y valoración de los elementos clave, se han utilizado cuestionarios para el alumnado y profesorado, y focus group virtuales entre el profesorado y la investigadora mediante el uso de Google Hangouts. Además, al finalizar cada fase de la IBD 
se ha realizado un focus group presencial de cierre entre los investigadores y el profesorado participante. Este focus group ha servido para valorar todo el trabajo realizado en cada fase y definir propuestas de mejora sobre la integración y evolución de los elementos clave para implementar en la siguiente fase.

Figura 5 - Instrumentos de la investigación

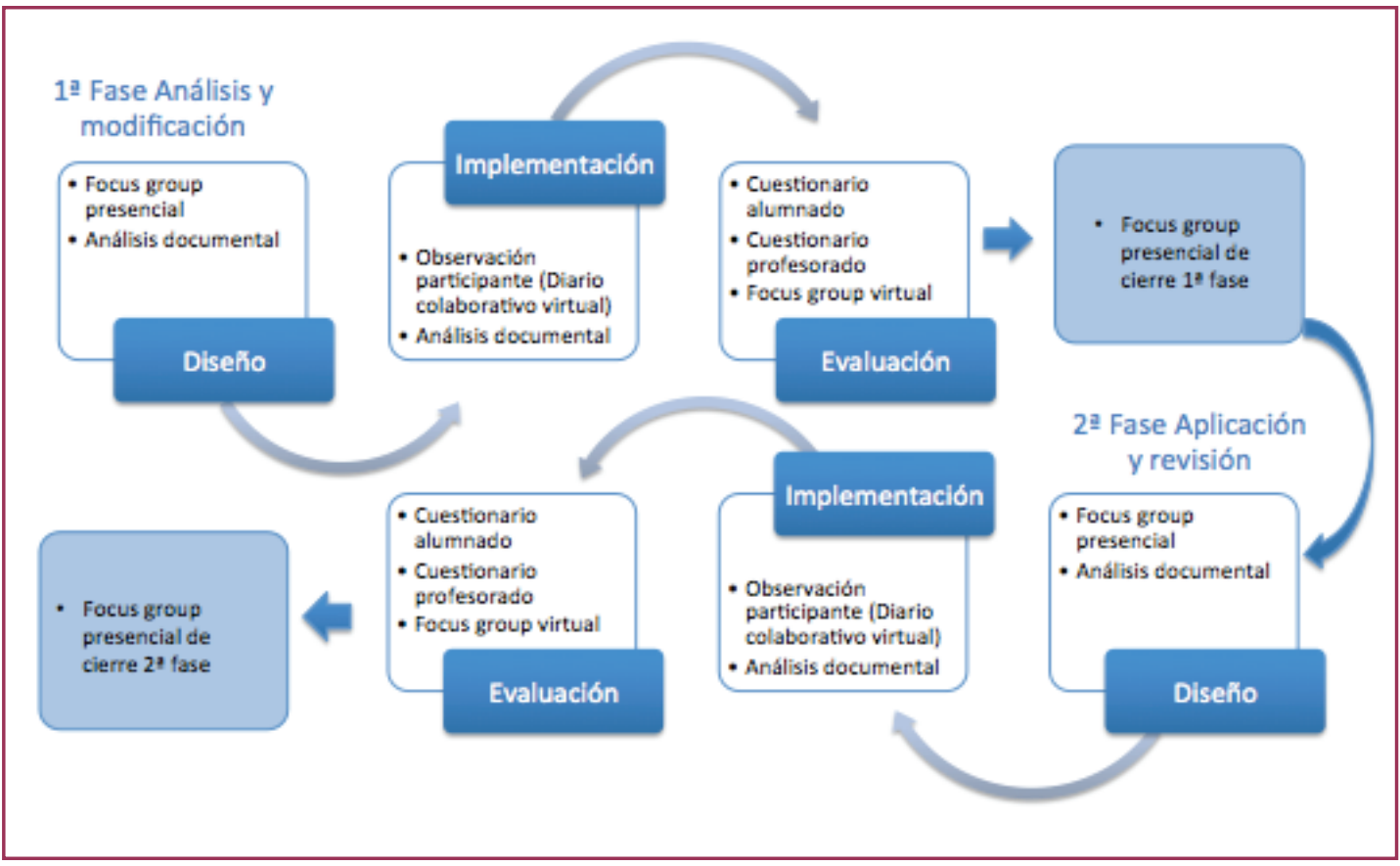

Fuente: Elaboración propia

\subsection{Análisis}

El hecho de haber llevado a cabo una IBD (GROS, 2012) como investigación cualitativa ha conllevado que el análisis de datos diera sentido a la información recogida, lo cual ha supuesto reducir e interpretar lo que las personas han dicho y lo que la investigadora ha visto, leído y observado a fin de construir significado (MERRIAM, 1998).

El análisis de datos se ha realizado atendiendo al carácter cíclico e iterativo de la IBD. De este modo, se han realizado dos tipos de análisis, el análisis retrospectivo al final de cada situación y cierre de cada fase de la IBD, y el análisis continuo fruto de las iteraciones cíclicas en el diseño, implementación y evaluación de las situaciones (GIBELLI, 2014; MOLINA et al., 2011).

El análisis continuo se ha realizado durante el diseño, implementación y evaluación de las situaciones mediante el uso del grupo de WhatsApp y ha tenido como objetivo obtener información de carácter práctico sobe los elementos clave a fin de anticipar, prever y dar feedback al profesorado para mejorar la implementación del diseño en el día a día en el aula, a la vez que se ha obtenido información relevante que más adelante se analizaría en el análisis retrospectivo que ha permitido evolucionar los elementos clave.

El análisis retrospectivo ha tenido como objetivo identificar los elementos clave para el diseño de situaciones de aprendizaje mediante el análisis de los datos recogidos a lo largo del proceso de investigación (MOLINA et al., 2011). En el presente estudio, el análisis retrospectivo 
se ha realizado al finalizar cada situación de aprendizaje, con lo cual en total, se han realizado seis análisis pertenecientes a las seis situaciones.

Para el análisis de datos se han seguido diferentes fases. En primer lugar, se ha recopilado información de los distintos instrumentos, a continuación, se han reducido los datos y se han establecido categorías de los elementos clave. Seguidamente, se ha seleccionado la información relevante para el nuevo diseño y se ha representado. Finalmente, se ha respondido a la pregunta del estudio.

\section{RESULTADOS Y DISCUSIONES}

\subsection{Elementos clave en la $1^{\text {a }}$ fase de la investigación}

La investigación ha partido de unos elementos clave fruto de la revisión de la literatura y de los documentos oficiales. Los elementos clave que se han definido en las situaciones de aprendizaje de la 1aㅡ fase de la investigación han sido: saludable, TIC, cooperación, real, transdisciplinariedad, retos, personalización, preguntas y motivaciones (PRENSKY, 2011; ACASO, 2013; IBARROLA, 2013; MORA, 2013; HERNANDO, 2015; VEGARA, 2015; COBO, 2016). A lo largo del estudio, y en base a la literatura y a las iteraciones realizadas entre la investigadora y el profesorado, se han analizado cuáles eran los elementos clave. Se han descartado algunos que han quedado absorbidos por otros como es el caso de las preguntas incluida en los retos, y la gamificación englobada en las motivaciones. Además, en la tercera situación de esta 1 a fase se ha considerado necesario incorporar por primera vez el elemento emociones (PELLICER, 2011) obteniendo una elevada presencia.

Figura 6 - Cuestionario de valoración del profesorado sobre el grado de presencia de los elementos clave en la tercera situación de la 1 a fase de la investigación

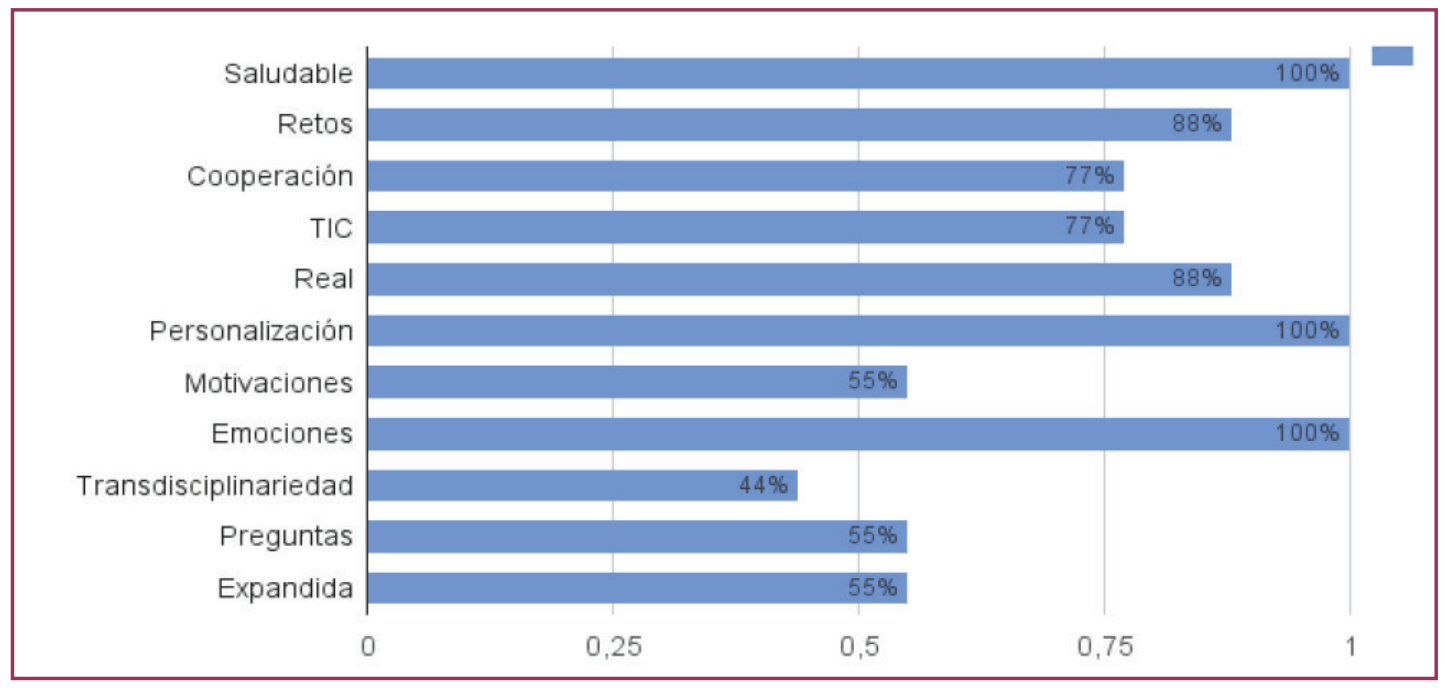

Fuente: Elaboración propia

Una vez realizado el análisis de la 1a fase de la investigación, se ha observado como los elementos clave se podían organizar en elementos curriculares, pedagógicos y personales, entendiendo las TIC como elemento integrador de todos ellos. Estos elementos se han implementado en las situaciones de aprendizaje de la $2^{a}$ fase de la investigación. 
Figura 7 - Elementos clave al finalizar la 1a fase de la investigación

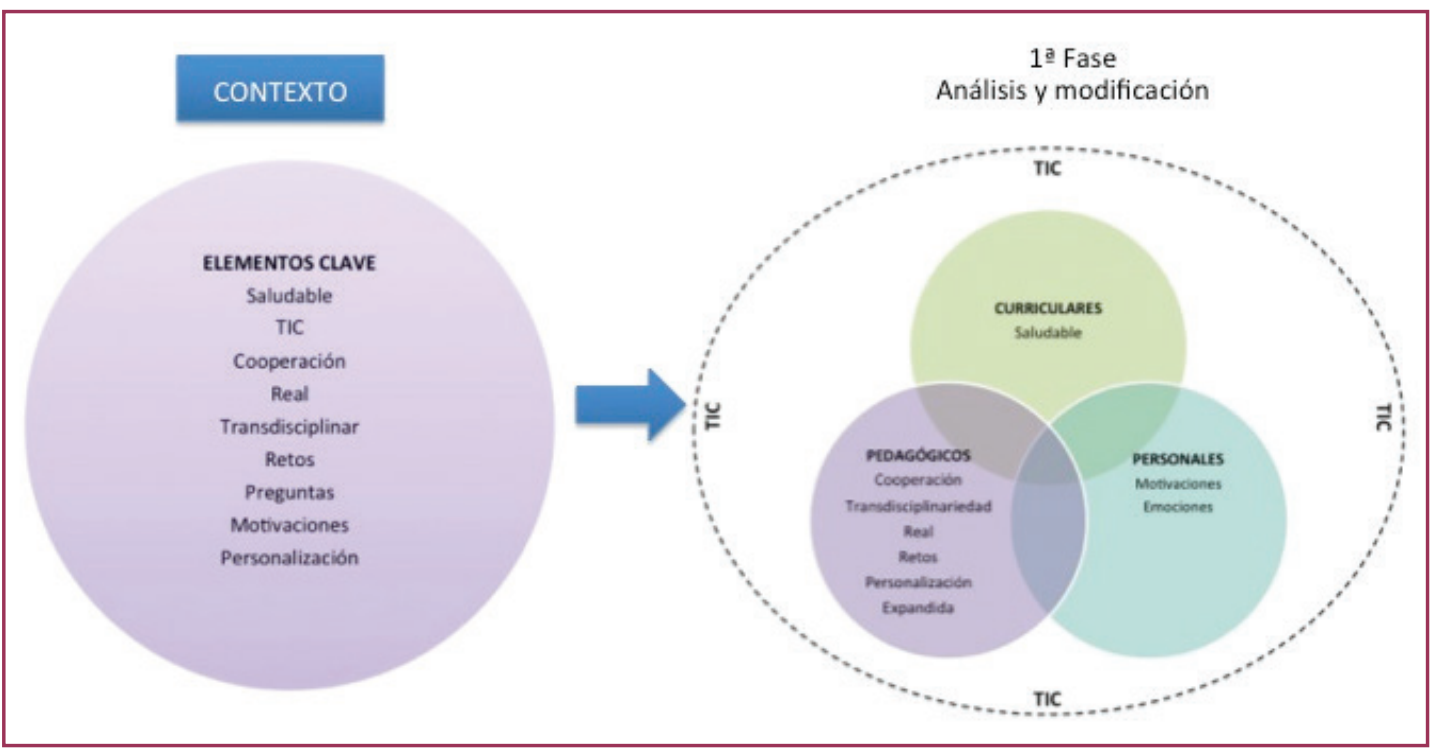

Fuente: Elaboración propia.

\subsection{Elementos clave en la $2^{a}$ fase de la investigación}

Los elementos de la $1^{\underline{a}}$ fase de la investigación nos han permitido evolucionar en la $2^{\underline{a}}$ fase: "Pienso que tenemos que hacer un paso atrás y hacer una redefinición de elementos. Estamos viendo que los elementos han dado buenos resultados" (Investigador M).

En esta línea, y en relación a los elementos personales, se ha constatado a lo largo del estudio como el juego, la música y la sorpresa han sido aspectos vinculados con la motivación y el aprendizaje del alumnado. De hecho, jugar, ha sido una demanda por parte del alumnado quien ha manifestado constantemente el deseo de jugar más: "Tener más tiempo para jugar al juego de nuestro grupo" (Alumno A). Las situaciones de aprendizaje de la investigación ya habían incorporado el juego y las actividades lúdicas como contenido curricular con la intención de desarrollar las cualidades físicas y el trabajo en equipo, aspecto muy bien acogido entre el alumnado: "Lo que más me ha gustado ha sido inventar un juego y jugar el juego de los otros" (Alumno B); "Lo que más me ha gustado ha sido la actividad de los juegos en grupo porqué me divertí mucho y trabajamos en equipo" (Alumno C). Sin embargo, observando las valoraciones del alumnado, en la $2^{\underline{a}}$ fase de la investigación se ha incluido al final del calentamiento un "juego de liberación" de elevada intensidad y motivación con la intención de dar al alumnado el espacio necesario para liberar su energía, potenciar la diversión y focalizar su atención hacia el aprendizaje posterior. Los resultados obtenidos han sido muy favorables, la motivación, implicación, atención y predisposición del alumnado por el aprendizaje posterior ha mejorado. Estos resultados siguen la línea de múltiples autores y estudios que evidencian el potencial del juego como herramienta de motivación y aprendizaje. Además, y en el caso específico de la Educación Física, el juego es un recurso didáctico de elevada carga motivacional que permite el desarrollo e integración de otros conocimientos (BAENA; RUIZ, 2016).

Otro aspecto motivacional que ha valorado positivamente el alumnado ha sido el uso de la música en clase: "Lo que más me ha gustado ha sido la música a la hora de hacer los tabatas porque motiva mucho" (Alumno D). En la misma dirección, el profesorado ha podido vivenciar la elevada carga motivacional que supone trabajar con música en el aula y así lo ha manifestado: "Poder trabajar con música genera un estado motivacional muy alto" (Profesor C). De este modo, 
la investigación valora muy positivamente el uso de la música en las sesiones de Educación Física como elemento motivacional que predispone y facilita el aprendizaje del alumnado.

Finalmente, el tercer aspecto que ha favorecido la motivación del alumnado ha sido introducir la sorpresa y lo inesperado en el aula (ACASO, 2013) aspectos que han permitido mantener la motivación del alumnado hacia el aprendizaje. De esta manera, algunas de las estrategias implementadas con éxito en las situaciones han sido introducir simulaciones mediante soporte visual, crear personajes ficticios para repartir los roles de equipo, aplicar dinámicas para descifrar códigos secretos y encontrar pistas para descubrir qué iba a suceder en clase, contextualizar las situaciones en base a historietas al estilo videojuego y utilizar las insignias como herramientas motivacionales propias del ámbito de la gamificación (ZICHERMANN; CUNNINGHAM, 2011). De este modo, concluimos que para favorecer la motivación del alumnado y despertar el interés hacia el aprendizaje, el juego, la música y la sorpresa son grandes herramientas a tener en cuenta en las situaciones.

En referencia al elemento TIC, la investigación ha demostrado su importancia en el diseño e implementación del estudio actuando como mediadora para la creación de una comunidad virtual de aprendizaje construyendo nuevas formas de enseñar, aprender y potenciar la mejora de la tarea docente (ROMEU, 2011).

Por otro lado, la investigación ha mostrado que la metodología ha avanzado de forma paralela con la tecnología. El uso de metodologías activas en las situaciones ha implicado la demanda y el uso de la tecnología para mejorar su implementación, amplificar las capacidades y propiciar nuevos saberes (COBO, 2016). De esta manera, la tecnología utilizada ha favorecido el uso de las metodologías activas centradas en el alumnado, potenciando la personalización y el aprendizaje colaborativo entre iguales (PRATS; OJANDO, 2015). Una constante observada en todas las situaciones de aprendizaje, ha sido la necesidad del uso de aplicaciones móviles en el aula de Educación Física para visibilizar los desafíos al alumnado, trabajar en base a retos y resolver determinados problemas. Atendiendo al carácter procedimental de la asignatura, el móvil se ha convertido en un gran aliado para el aprendizaje y para la incentivación de la práctica física en Educación Física (MONGUILLOT et al., 2013). Fruto del análisis realizado en la investigación, consideramos que el uso adecuado e intencionado de las tecnologías abren las puertas y amplifican las oportunidades de enseñanza y aprendizaje de acorde a las demandas sociales del momento (ADELL; CASTAÑEDA, 2013).

Cuanto a los elementos pedagógicos, la investigación ha demostrado la importancia de integrar diferentes estrategias metodológicas y de evaluación basadas en las metodologías activas y pedagogías emergentes a fin de potenciar competencias del siglo XXI como la resolución de problemas mediante la negociación, el pensamiento crítico y la comunicación eficaz mediante el trabajo en equipo (GIJSBERS; VAN SCHOONHOVEN, 2012).

Referente al elemento curricular, el enfoque metodológico de la Educación Física en las situaciones de aprendizaje, se ha caracterizado por ser saludable, cooperativo, funcional, personalizado, tecnológico y expandido. En esta línea, un alumno manifestaba: "Lo que más me ha gustado ha sido trabajar en equipo porque colaborabas" (Alumno F). De esta manera se ha impulsado una Educación Física basada en situaciones reales que atienden a los intereses del alumnado y donde éste pueda desarrollar habilidades cognitivas de orden superior (CHURCHES, 2009).

De este modo, al finalizar la $2^{\underline{a}}$ fase del estudio los elementos clave han quedado organizados en 4 cuatro dimensiones: tecnológica, pedagógica, curricular y personal, dando 
lugar al modelo TPACKPEC que integra la dimensión personal con los elementos emociones y motivaciones como conocimiento fundamental en el proceso de aprendizaje.

Además de las cuatro dimensiones de conocimiento, el modelo TPACKPEC incluye el elemento transdisciplinariedad puesto que el carácter colaborativo, abierto y flexible de las situaciones de aprendizaje del estudio invitan y abren las puertas a futuras relaciones y sinergias con otras materias curriculares. Esta característica de las situaciones, es un aspecto fundamental si tenemos en cuenta que el currículo competencial demanda el trabajo globalizado mediante proyectos transdisciplinares a fin de desarrollar las competencias del alumnado (TRUJILLO, 2016).

\section{CONCLUSIONES}

En respuesta a la pregunta guía y tal como se ha ido evidenciando a lo largo del estudio, los elementos clave necesarios para diseñar una situación de aprendizaje mediada por TIC en Educación Física se han clasificado en 4 dimensiones de conocimiento: tecnológica, pedagógica, curricular y personal, dando lugar al modelo TPACKPEC. Como ya se ha dicho, esta organización parte del modelo TPACK (KOEHLER; MISHRA, 2009) y hace un paso más allá integrando la dimensión personal con los elementos de emociones y motivaciones. Esta dimensión se origina en las aportaciones de la neurociencia al campo educativo que enfatizan la importancia de atender a las emociones, encender la curiosidad y crear un clima de aula favorable para la mejora del aprendizaje. Las emociones son la puerta de entrada al aprendizaje facilitándolo o dificultándolo con lo cual es fundamental tenerlas en cuenta. En esta línea, la dimensión personal aboga por concienciar al alumnado de sus fortalezas y debilidades, y fomentar el bienestar personal y social a fin de mejorar el proceso de aprendizaje (MORA, 2013; IBARROLA, 2013; PELLICER, 2015).

Figura 8 - Modelo TPACKPEC

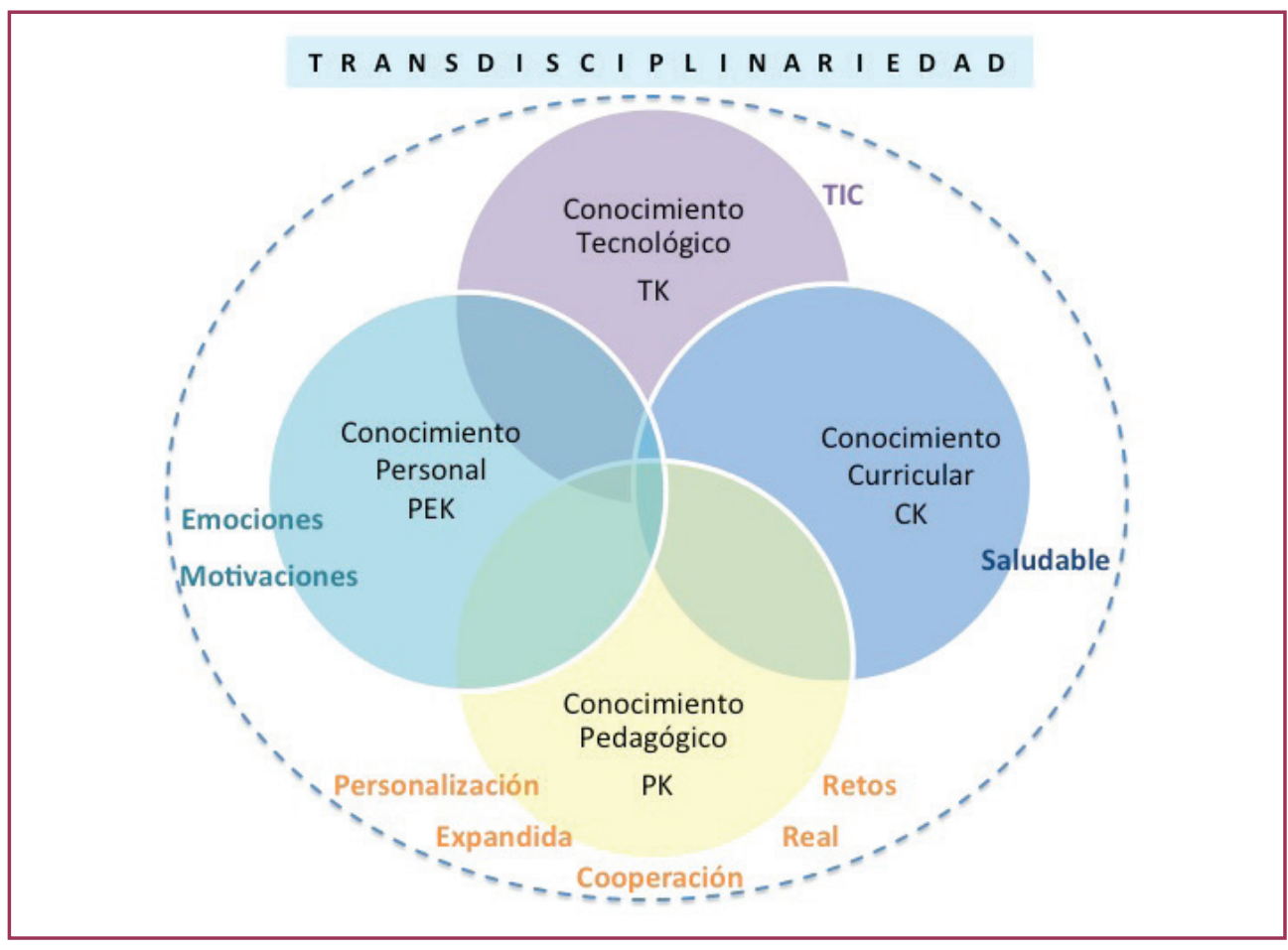

Fuente: Elaboración propia. 
Cuanto a la dimensión tecnológica y a la integración de las TIC en el aula, las conclusiones del estudio visibilizan la necesidad de amplificar y expandir el aula de Educación Física de la mano de la tecnología. Para ello, el m-learning se perfila como una excelente herramienta tecnológica en clase de Educación Física gracias a su portabilidad, uso generalizado (CANTILLO et al., 2012) y a las distintas aplicaciones que permiten monitorizar la actividad física.

En relación a la dimensión curricular, la investigación demuestra la necesidad de un cambio de enfoque metodológico de la materia. Una Educación Física centrada en el desarrollo de la salud, personalizada, cooperativa, útil y real, capaz de desarrollar las habilidades del siglo XXI y de expandir el aula ofreciendo nuevas oportunidades de aprendizaje.

En referencia a la dimensión pedagógica las situaciones del estudio abogan por la implementación de las metodologías activas y de la evaluación consciente del alumnado como herramientas para la transformación educativa. En esta línea, el uso del aprendizaje cooperativo, la gamificación, el aprendizaje basado en retos junto a una evaluación formativa, reguladora y auténtica que potencie la autoevaluación y coevaluación se convierten en herramientas fundamentales para el cambio educativo (SANMARTí, 2010; VERGARA, 2015; HERNANDO, 2015).

Otra de las conclusiones del estudio demuestra la importancia del trabajo colaborativo entre docentes como herramienta para la construcción colaborativa de conocimiento y mejora profesional. La participación activa y colaborativa del profesorado en la investigación mediante el uso de la IBD ha posibilitado aprender, innovar y mejorar la tarea docente (AMIEL; REEVES, 2008). La IBD ha permitido investigar, crear y ampliar el conocimiento sobre el diseño e implementación de situaciones de aprendizaje innovadoras. Las interacciones realizadas por el profesorado durante las implementaciones, han facilitado la obtención de conocimiento para la mejora de la práctica educativa a fin de crear modelos útiles para el diseño de entornos de aprendizaje (GROS, 2012).

Para finalizar, y como posibles líneas de investigación, planteamos el modelo propuesto con los elementos clave y su transferencia a otras materias o escenarios educativos.

\section{REFERENCIAS}

ACASO, María. rEDUvolution: Hacer la revolución en la educación.Madrid: Paidós, 2013.

ADELL, Jordi; CASTAÑEDA, Linda. El ecosistema pedagógico de los PLEs. En: CASTAÑEDA, Linda; ADELL, Jordi (Eds.). Entornos Personales de Aprendizaje: Claves para el ecosistema educativo en red. Alcoy: Marfil. 2013. p. 29-51.

AMIEL, Tel; REEVES, Thomas C. Design-Based Research and Educational Technology: Rethinking Technology and the Research Agenda. Educational Technology \& Society, v. 11, n. 4, p. 29 - 40, 2008.

BAENA, Antonio; RUIZ, Pedro Jesús. El juego motor como actividad física organizada en la enseñanza y la recreación. EmásF Revista Digital de Educación Física, v.7, n.38, p.73-86, 2016.

CABERO, Júlio (Coord.). La formación del profesorado en TIC: Modelo TPACK. Sevilla: 
CANTILLO, Carmen; ROURA, Margarita; SÁNCHEZ, Ana. Tendencias actuales en el uso de dispositivos móviles en educación.La Educ@ción Digital Magazine, v.147, p.1-20, jun. 2012.

CAPLLONCH BUJOSA, Marta. Las tecnologías de la información y la comunicación en la Educación Física de Primaria: Estudio de sus posibilidades educativas.329 f. 2005. Tese (Doctoral) Universidad de Barcelona, Barcelona, 2005.

CHURCHES, Andrew. Taxonomía de Bloom para la era digital. Eduteka. 2009. Disponível em:<http:// eduteka.icesi.edu.co/articulos/TaxonomiaBloomDigital>. Acceso en: 20 ago. 2018.

COBO,Cristóbal. La Innovación Pendiente: Reflexiones (y Provocaciones) sobre educación, tecnología y conocimiento. Montevideo: Penguin Random House, 2016. (Colección Fundación Ceibal/ Debate).

DECRET 143/2007, de 26 de juny, pel qual s'estableixl'ordenaciódelsensenyaments de l'educaciósecundàriaobligatòria. Diari oficial de la Generalitá de Catalunya, n. 4915, seció: Disposiciones, p. 21870, 29 jun.2007.

DURALL, Eva et al. Perspectivas tecnológicas: educación superior en Iberoamérica 2012-2017. Austin: New Media Consortium, 2012.

FERRERES, Carlos. La integración de las tecnologías de la información y de la comunicación en el área de la educación física de secundaria: análisis sobre el uso, nivel de conocimientos y actitudes hacia las tic y de sus posibles aplicaciones educativas. 787f. 2011. Tesis (Doctoral) Universitat Rovira i Virgili, Tarragona, 2011.

GALLEGO, Vanesa et al.. La orientación en el medio natural: aprendizaje ubicuo mediante el uso de la tecnología. Movimento, v.23, n.2, p.755-770, abr/jun. 2017.

GARCÍA, José Ángel. Modelo educativo basado en competencias: Importancia y necesidad. Revista Electrónica Actualidades Investigativas en Educación", v.111, n.3, p.1-24, 2011.

GIJSBERS, Govert; VAN SCHOONHOVEN, Bas. The future of learning: a foresight study on new ways tolearn new skills for future jobs. European Foresight Platform (EFP) Brief, n.222, 2012.

GONZÁLEZ, Carles; MONGUILLOT, Meritxell; ZURITA, Carles. Una Educación Física para la vida. Barcelona: Inde. 2014.

GONZÁLEZ, Carles et al. Physical education and ICT: and unstoppable combination. In: NOVAK, Dario et al. (Eds.). Physical Education and New Technologies. Zagreb: Croatian KinesiologyAssociation, 2016. p. 61-70.

GROS, Begoña. Retos y tendencias sobre el futuro de la investigación acerca del aprendizaje con tecnologías digitales. RED. Revista de Educación a Distancia, n. 32, 2012. Disponible em:< https:// www.um.es/ead/red/32/gros.pdf>. Acceso em: 20 ago.2018

GIBELLI, Tatiana. La investigación basada en diseño para el estudio de una innovación en educación superior que promueve la autorregulación del aprendizaje utilizando TIC. In: CONGRESO IBEROAMERICANO DE CIENCIA, TECNOLOGÍA, INNOVACIÓN Y EDUCACIÓN.1. 2014. Anais... Buenos Aires:2014. Articulo 1440.

HERNÁNDEZ, Núria. El trabajo colaborativo en entornos virtuales de educación superior. $769 f$. 2015. Tesis (Doctoral) -. Universidade da Coruña, La Coruña, 2015.

HERNANDO, Alfredo. Viaje a la escuela del siglo XXI: así trabajan los colegios más innovadores del mundo. Madrid: Fundación Telefónica, 2015. 
IBARROLA, Begoña. Aprendizaje emocionante: Neurociencia para el aula. Madrid: Ediciones SM, 2013.

KOEHLER, Matt; MISHRA, Punnya. What is Technological Pedagogical Content Knowledge (TPACK)? Contemporary Issues in Technology and Teacher Education, v.9, n.1, p. 60-67, 2009.

LLEIXÀ, Teresa. Educación física y competencias básicas. Contribución del área a la adquisición de las competencias básicas del currículum. Tándem. Didáctica de la EducaciónFísica, v. 23, p. 31-37, 2007.

MERRIAM, Shara, B. Case Study Research in education: a Qualitative Approach. San Francisco: Jossey-Bass, 1998.

MOLINA, Marta et al. Un acercamiento a la investigación de diseño a través de los experimentos de enseñanza. Enseñanza de las ciencias: revista de investigación y experiencias didácticas, v.29, n.1, p. 75-88, 2011.

MONGUILLOT, Meritxell; GUITERT, Montse; GONZÁLEZ, Carles. El trabajo colaborativo virtual: herramienta de formación del profesorado de educación física. Retos: nuevas tendencias en educación física, deporte y recreación, v.24, p. 24-27, 2013.

MONGUILLOT, Meritxell et al. Mobile learning: una experiencia colaborativa mediante códigos QR. Revista de Universidad y Sociedad Del Conocimient, v.11, n.1, 2014. Disponible em: < https:// www.raco.cat/index.php/RUSC/article/viewFile/285043/373040> Acceso em: 20 ago.2018.

MONGUILLOT, Meritxell; GONZÁLEZ, Carles; GUITERT, Montse. El whatsapp como herramienta para la colaboración docente. EmásF Revista Digital de Educación Física, v. 44, n.8, p.56-62, 2017.

MORA, Francisco. Neuroeducación: Solo se aprende aquello que se ama. Madrid: Alianza Editorial, 2013.

ORGANIZACIÓN MUNDIAL DE LA SALUD. Recomendaciones mundiales sobre actividad física para la salud. Ginebra, 2010.

PELLICER, Irene. Educación física emocional: de la teoría a la práctica. Barcelona: Inde. 2011.

PELLICER, Irene. Neuro EF: la revolución de la educación física desde la neurociencia. Barcelona: Inde, 2015.

PIÉRON, Maurice; RUIZ JUAN, Francisco; GARCÍA MONTES, María Elena. La opinión del alumnado de enseñanza secundaria sobre las clases de educación física: un desafió para los profesores y formadores. Revista Fuentes, n.8, p.159-175, 2008.

PRAT, Queralt; CAMERINO, Oleguer. Introducción de las TIC en educación física: Estudio descriptivo sobre la situación actual. APUNTS. Educació Física i L'esport, n.113, p. 37- 44, 2013.

PRATS, Miquel Ángel; OJANDO, Elena Sofía. ¿ Pueden las TIC mejorar los resultados académicos? Diseños formativos y didácticos con soporte TIC que mejoran los aprendizajes: el caso de los contenidos digitales de ortografía de Digital-Text. Educatio Siglo XXI, v.33, n.3, p.85-102, 2015.

PRENSKY, Marc. Enseñar a nativos digitales. Madrid: Ediciones SM, 2011.

RINAUDO, María Cristina; DONOLO, Danilo. Estudios de diseño: una perspectiva prometedora en la investigación educativa. RED Revista de Educación a Distancia, v. 22, 2010. Disponible em: < http:// revistas.um.es/red/article/view/111631/105951>. Acceso em: 20 ago.2018. 
ROMEU, Teresa. La docencia en colaboración en contextos virtuales: estudio de caso de un equipo de docentes del área de competencias digitales de la UOC. 351 f. 2011. Tesis (Doctoral) Universidad Oberta de Catalunya. Barcelona, 2011.

SALINAS, Jesús; DE BENITO, Bárbara; LIZANA, Alexandra. Competencias docentes para los nuevos escenarios de aprendizaje. Revista interuniversitaria de formación del profesorado, v.79, p.145163, 2014.

SANMARTÍ, Neus. Avaluar per aprendre: L'avaluació per millorar els aprenentatges de l'alumnat en el marc del currículum per competències. Barcelona: Generalitat de Catalunya. Departament d’Educació. Direcció General de l'Educació Bàsica i el Batxillerat, 2010.

TIRADO, Miguel Ángel. L'actitud crítica sobre el culte al cos des de l'Educació Física. 430f. 2010. Tesis (Doctoral) - Universitat de les Illes Balears, Palma, 2010.

TRUJILLO SAÉZ, Fernando. El diseño de proyectos y el currículo. Cuadernos de Pedagogía, n.472, p.66-69, 2016.

VALVERDE, Jesús; GARRIDO, Mari Carmen; FERNÁNDEZ, Rosa. Enseñar y aprender con tecnologías: un modelo teórico para las buenas prácticas educativas con TIC. Teoría de la Educación, v.11, n.3, p. 203-229, 2010.

VERGARA, Juan José. Aprendo porque quiero: el Aprendizaje Basado en Proyectos (ABP), paso a paso. Madrid: Ediciones SM, 2015.

ZICHERMANN, Gabe; CUNNINGHAM, Christopher.Gamification by design: Implementing game mechanics in web and mobile apps. Sebastopol: O'Reilly, 2011. 
